ज્પ FRANÇAISE

$>\mathrm{DE}$

될 PÉDAGOGIE

\section{Revue française de pédagogie}

Recherches en éducation

156 | juillet-septembre 2006

Les espaces locaux d'interdépendance entre

établissements : une comparaison européenne

\title{
Attractivité et différenciation des sites universitaires : une analyse de cas en Aquitaine
}

Attractivity and differenciation of colleges: analysing a case in Aquitaine, France Atractivo y diferenciación de las áreas universitarias : un análisis en Aquitania Anziehungskraft und Differenzierung der universitärischen Polen: Analyse eines Falls in der Aquitaine

\section{Georges Felouzis}

\section{OpenEdition}

Journals

Édition électronique

URL : http://journals.openedition.org/rfp/598

DOI : 10.4000/rfp.598

ISSN : 2105-2913

Éditeur

ENS Éditions

Édition imprimée

Date de publication : 1 juillet 2006

Pagination : 101-116

ISBN : 978-2-7342-1060-3

ISSN : 0556-7807

Référence électronique

Georges Felouzis, «Attractivité et différenciation des sites universitaires : une analyse de cas en Aquitaine », Revue française de pédagogie [En ligne], 156 | juillet-septembre 2006, mis en ligne le 27 septembre 2010, consulté le 19 avril 2019. URL : http://journals.openedition.org/rfp/598 ; DOI : $10.4000 /$ rfp.598 


\section{Attractivité et différenciation des sites universitaires: une analyse de cas en Aquitaine}

Georges Felouzis

L'article propose une réflexion sur la territorialisation de l'enseignement supérieur du point de vue des parcours étudiants. II considère l'ensemble des étudiants primo entrants en 1997 dans un site universitaire en Aquitaine, ce qui correspond à environ 11800 étudiants. II propose un ensemble d'indicateurs (nombre théorique d'étudiants par site, taux de recrutement local, taux de rétention locale et taux d'étudiants hors académie) dont les variations en fonction du site d'étude et de la filière montrent trois faits essentiels. 1. L'attractivité des sites dépend de facteurs très variés tels que le statut (délocalisé ou de plein exercice), la situation géographique, la taille et la discipline enseignées. 2. Cette attractivité a des conséquences sur la nature du public de chaque site et filières, mesurée par la proportion d'étudiants à l'heure, de bacheliers généraux et de milieu cadre. 3. La nature de l'offre de formation dans une discipline donnée en Aquitaine influe nettement sur la composition sociale et académique des différents sites. La comparaison entre le public "attendu » et le public " observé » montre de nettes différences qui reflètent une division du travail entre les sites centraux et leurs antennes délocalisées.

Descripteurs (TEE) : Aquitaine, étudiants, offre de formation, universités.

es politiques d'enseignement supérieur depuis la L fin des années 1980 ont orienté le développement universitaire vers une territorialisation croissante des premiers cycles (Filâtre, 2003). Ce phénomène correspond d'abord à l'émergence des collectivités territoriales comme acteur à part entière des politiques universitaires. Non pas seulement comme bailleurs de fonds, mais aussi comme acteurs animés d'une volonté pour, entre autres, susciter un développement local. Cette territorialisation s'est concrétisée par le développement de nouveaux sites universitaires délocalisés. Le « maillage territorial » est aujourd'hui d'une densité sans précédent en France. Les capitales régionales n'ont plus le monopole des formations supérieures, et les villes moyennes accueillent pour la plupart des formations de premier cycle universitaire, d'IUT, voire des formations de niveau Master (France, 2003).

Cette territorialisation de l'enseignement supérieur est le fruit de processus multiples qui doivent autant à la politique de décentralisation, qu'aux plans 
«Université 2000 » et « U3M » et à la volonté de l'État de créer des «systèmes régionaux d'enseignement supérieur ». Ce processus a abouti à la création progressive d'un "service public de proximité " d'enseignement supérieur, favorisant l'accès à l'université, plus ouverte aux enfants issus de milieux modestes (Bourdon et al., 1994). L'une des fonctions des délocalisations est donc de démocratiser l'accès à l'enseignement supérieur, et de ce point de vue le pari est réussi. Les sites implantés dans les villes moyennes attirent un public socialement moins favorisé que les sites centraux et scolairement moins performant. Ils contribuent ainsi à favoriser la poursuite d'étude dans le supérieur et recrutent de nouveaux publics pour les universités. On peut ajouter à ce premier point qu'en termes de probabilités de réussite, beaucoup de sites délocalisés n'ont pas à rougir de leurs performances, tant il est vrai que la réussite peut y être équivalente à celle des sites centraux (Felouzis, 2001a ; Michaut, 2005).

La territorialisation de l'enseignement supérieur a suscité de larges courants de recherche en politiques publiques qui se proposent d'analyser les processus de construction de l'offre de formation locale, le poids des différents acteurs institutionnels, les évolutions du rôle des collectivités locales, etc. Elle a aussi suscité, mais dans une moindre mesure, des réflexions sur les conséquences de ces délocalisation sur les acteurs de l'université, qu'il s'agisse des universitaires (Losego, 2004), de la recherche (Grossetti, 1995), ou encore des parcours étudiants (Felouzis, 2001b). L'une des questions que l'on se pose aujourd'hui sur les sites délocalisés est celle de leur avenir dans le contexte actuel de baisse des effectifs universitaires. À la fin de la décennie 1980, le nombre d'étudiants ne cesse d'augmenter. Le taux de croissance annuel frôle les $10 \%$ et les sites délocalisés ont été une des solutions envisagées pour " désengorger " les universités massifiées des grandes métropoles régionales. Aujourd'hui, la tendance s'est inversée. Dans la plupart des grandes disciplines, le nombre d'étudiants a baissé de manière significative depuis la rentrée charnière de 1995. En Sciences, les effectifs universitaires (hors IUT) passent de plus de 63000 à la rentrée 1995 à 32000 à la rentrée 2004 (- $50 \%$ environ), en droit la baisse des effectifs est de $22 \%$, et de $14 \%$ environ en Lettres et Sciences humaines, en Sciences économiques et AES (France, 2004). Les sites délocalisés, qui font aujourd'hui pleinement partie du paysage universitaire de nos régions, s'en trouvent fragilisés. D'abord parce que le nombre de leurs étudiants tend à baisser. Ensuite parce que la complémentarité dans la gestion des flux étudiants qui les unissaient à leur université de référence peut facilement devenir une concurrence dans un contexte de baisse des effectifs universitaires.

Il devient alors pertinent de se questionner sur les effets de ce nouveau contexte de concurrence entre les sites universitaires régionaux, et ainsi comprendre comment se construit l'attractivité des sites, comment les flux étudiants s'orientent vers tel ou tel site, et quelles sont les conséquences de ces choix sur la composition académique et sociale des premiers cycles universitaires. Les effets de site à l'université, que l'on mesure habituellement en fonction des taux d'accès à tel ou tel niveau de certification universitaire (Felouzis, 2000), se conçoivent ici en fonction de l'attractivité et de ses effets sur la nature de leur public en premier cycle. Sur l'ensemble de l'Aquitaine, certaines disciplines sont enseignées sur plusieurs sites dont le statut est contrasté (de plein exercice ou délocalisé), d'autres ne sont enseignées que dans des sites de plein exercice, d'autres enfin sont le monopole d'un site unique, par exemple la médecine à Bordeaux.

Dans ce foisonnement de possibilités, quels sont les sites qui peuvent être définis comme "attractifs " et ceux qui le sont moins? Les données dont nous disposons sont issues d'une recherche sur les effets d'établissement à l'université (1) dont la base empirique est une cohorte d'étudiants primoentrants dans une formation universitaire en Aquitaine en 1997. La recherche a porté sur les taux d'accès en second cycle « toutes choses égales par ailleurs " en fonction du site. Cela représente plus de 11800 étudiants inscrits soit en premier cycle, soit en IUT. Sur ces 11800 étudiants, certains choisissent une filière enseignée dans une seule université et un seul site. Les études médicales sont dans ce cas, ainsi que la pharmacie. Cela signifie que les étudiants n'ont pas le choix du site en Aquitaine et que seule une inscription à Bordeaux est possible pour eux. Dans d'autres cas, le choix du site est rendu possible par une territorialisation de certaines filières, même si les contraintes liées au lieu d'habitation ne sont pas à négliger. Le fichier SISE (2) ne considère que les étudiants inscrits dans une formation universitaire, ce qui exclu de fait les bacheliers inscrits en classes préparatoire, BTS ou encore dans des formations ne dépendant pas de l'enseignement supérieur universitaire. Le propos de cet article n'est donc pas d'étudier les orientations post-baccalauréat en général, car dans ce cas, les données disponibles ne rendraient pas compte de l'ensemble des choix possibles: un bachelier peut en effet ne pas 
s'inscrire dans l'enseignement supérieur, s'orienter vers une grande école, une école de commerce ou encore vers la préparation d'un BTS. II s'agit plutôt de nous questionner sur le choix du site d'étude par les étudiants inscrit dans une discipline donnée, et ses conséquences éventuelles sur la nature du public de chaque site.

Dans la réalité toutefois, le champ des possibles est bien plus étendu. Un étudiant peut choisir d'étudier dans une autre académie que celle où il a passé son baccalauréat. C'est là aussi une des limites de nos données. Nous ne pouvons que partiellement évaluer les transferts inter académiques lors de la première inscription. Le nombre d'étudiants inscrits en Aquitaine en première année et venant d'une autre académie nous est connu, mais le nombre de bacheliers aquitains s'inscrivant dans une autre académie nous échappe. On peut donc considérer que nous disposons de données permettant d'approcher la question du choix du site de façon à raisonner sur des étudiants inscrits dans une des disciplines universitaires en Aquitaine. On peut ainsi approcher le public "naturellement » dévolu à chaque site, et le public « réellement » inscrit.

\section{LE CHOIX DU SITE : ÉLÉMENTS DE MÉTHODE}

Contrairement à l'enseignement secondaire, pour lequel les établissements sont "sectorisés", c'està-dire qu'ils recrutent dans un secteur prédéfini, les sites universitaires ne sont en rien limités dans leurs modalités de recrutement. Pour comprendre les modalités et les conséquences du choix du site par les étudiants, il nous faut construire un critère permettant d'évaluer le public susceptible de s'inscrire dans chaque site universitaire. Ce critère est construit à partir de la résidence d'origine des étudiants, c'està-dire par le domicile des parents. Ce domicile est défini ici en fonction du département. Cinq départements composent l'académie: La Dordogne (24), la Gironde (33), les Landes (40), le Lot-et-Garonne (47) et les Pyrénées-Atlantiques (64).

On peut donc définir le public « naturel » de chaque site en fonction de son département. La comparaison de ce «public attendu " avec la réalité observée donne une mesure des choix d'inscription et de leurs conséquences en termes de composition sociale et scolaire des sites en première année. C'est ainsi que le nombre théorique d'étudiants est construit en considérant simplement le "bassin d'étudiants" du département pour chaque site et discipline. La comparaison avec le nombre réel d'inscrits permet d'apprécier l'attractivité des sites. Toutefois, ces indicateurs ne peuvent être lus de façon " directe ", car tous les départements ne proposent pas un enseignement dans toutes les disciplines, et d'autres proposent les mêmes formations dans deux sites différents. II faudra donc se garder d'interpréter un « gain " ou une «perte » d'étudiants par rapport à la situation théorique comme on interprèterait le même phénomène en comparant des établissements d'enseignement secondaire. Par exemple en Économie et Gestion dans les Pyrénées-Atlantiques, deux sites proposent un enseignement ( $\mathrm{Pau}$ et Bayonne). Nécessairement, chaque site ne fera pas "le plein " des étudiants du département. Mais l'on sait par ailleurs que les étudiants des Landes ou du Lot-etGaronne peuvent très bien choisir l'un de ces sites. Ce qui nous conduit à raisonner en termes de taux de recrutement local pour un site et une filière donnée. II s'agit de la proportion d'étudiants de première année d'un site et d'une discipline dont les parents sont domiciliés dans le même département que le site d'étude. De même, le taux de rétention locale correspond, pour une discipline donnée, à la proportion d'étudiants d'un département inscrits dans le site de leur département. Enfin, le taux d'étudiants hors académie est aussi un indicateur, plus classique, d'attractivité d'un site.

Tableau I. - Sciences économiques et gestion.

Nombre d'étudiants «théorique » et « observé » en Aquitaine

\begin{tabular}{|l|c|c|c|c|}
\hline & $\begin{array}{c}\text { Nombre d'étudiants } \\
\text { théorique }\end{array}$ & $\begin{array}{c}\text { Nombre d'étudiants } \\
\text { observé }\end{array}$ & Différence brute & Différence relative \\
\hline Bordeaux & 251 & 419 & +168 & $+67 \%$ \\
\hline Bayonne & 133 & 60 & -73 & $-55 \%$ \\
\hline Pau & 133 & 105 & -28 & $-21 \%$ \\
\hline
\end{tabular}


Tableau II. - Sciences économiques et gestion.

Site d'étude en fonction du département de résidence des parents

\begin{tabular}{|c|c|c|c|c|}
\hline $\mathrm{Nb}$ & \multicolumn{4}{|c|}{ Site d'étude } \\
\hline Département des parents & Bayonne & $\begin{array}{l}\text { «Bordeaux } \\
\text { campus }\end{array}$ & Pau & Total \\
\hline Dordogne & & 33 & & 33 \\
\hline Gironde & & 249 & 2 & 251 \\
\hline Hors académie & & 57 & 13 & 70 \\
\hline Inconnu & 2 & 12 & 1 & 15 \\
\hline Landes & 7 & 24 & 21 & 53 \\
\hline Lot-et-Garonne & & 28 & 2 & 30 \\
\hline Pyrénées-Atlantiques & 51 & 16 & 66 & 133 \\
\hline Total & 30 & 419 & 105 & 584 \\
\hline
\end{tabular}

Un exemple permettra d'éclairer la nature de ces indicateurs. Les Sciences économiques et Gestion rassemblent en Aquitaine en 1997, 584 étudiants primo-entrants. Trois sites dispensent un enseignement de premier cycle dans cette discipline. II s'agit de «Bordeaux campus » (3), Bayonne et Pau.

Le nombre théorique d'étudiants à Bordeaux est de seulement 251: c'est le nombre d'inscrits dans cette discipline dont les parents habitent le département de la Gironde. Toutefois, nous en avons 419 qui s'inscrivent réellement, car certains viennent d'autres départements qui ne proposent pas cette formation en Aquitaine, d'autres viennent d'une autre académie. Pour Bayonne et Pau, le cas est différent puisque dans le seul département des PyrénéesAtlantiques, ces deux sites proposent des Sciences économiques et de la Gestion. Le nombre théorique d'étudiants pour chacun de ces sites est identique (c'est le nombre d'étudiants inscrits en Sciences économiques dont les parents sont domiciliés dans les Pyrénées-Atlantiques), et le nombre réel d'inscrits plus faible puisqu'ils se «partagent » ces étudiants.

Que nous apprennent ces indicateurs? D'abord que Bordeaux «draine " un nombre important d'étudiants hors de son département. La capitale régionale joue ici son rôle d'attraction d'autant plus fort que la Dordogne, les Landes et le Lot-et-Garonne ne proposent aucune formation de premier cycle en Sciences économiques. Pour $\mathrm{Pau}$ et le site délocalisé de Bayonne, on observe que le département attire plus d'étudiants (165) que son potentiel théorique (133).
Pau, site de plein exercice, attire probablement plus d'étudiants que Bayonne car il « perd » moins d'étudiants qu'il ne devrait, comparativement à Bayonne $(-21 \%$ contre $-55 \%)$.

Cela nous conduit à questionner l'origine géographique de ces étudiants. On donne dans le tableau II leur distribution en fonction du domicile des parents et du lieu d'étude.

Le site de Bordeaux campus rassemble 419 étudiants primo entrants dont 249 ont des parents qui habitent en Gironde. Cela représente un taux de recrutement local de $59,4 \%$. Les autres étudiants viennent soit d'un autre département de l'académie (101 étudiants), soit l'adresse de leurs parents est inconnue (douze cas), soit d'une autre académie (57 étudiants, ce qui représente $13,6 \%$ de l'ensemble). Si l'on considère maintenant le nombre d'étudiants girondins inscrit dans cette discipline, ils sont au nombre de 251. Et sur ces 251 étudiants, 249 s'inscrivent à Bordeaux. Le taux de rétention locale est de 249/251, ce qui aboutit à un taux de 99,2\%. On peut donc dire, à la lecture de ces premiers chiffres, que Bordeaux est « attractif " en Sciences économiques, comparativement aux autres sites dispensant cet enseignement en Aquitaine. Dans le cas de Pau et Bayonne, les deux sites se partagent les étudiants du département. De ce fait, le site délocalisé de Bayonne a un taux de rétention locale de $38 \%$ et Pau de $50 \%$.

On voit donc que ces quelques indicateurs nous informent sur l'attractivité des sites et l'usage qu'en 
font les étudiants. Mais au-delà de ces premiers résultats, on peut raisonner sur les conséquences de cette attractivité différentielle sur la nature du public de chaque site. Pour cela il nous faut établir une comparaison entre le public que l'on devrait trouver dans chaque site si chaque étudiant s'inscrivait dans son département (le public attendu) et son public réel. Soulignons que le public attendu n'est qu'une vue de l'esprit, car certains départements n'offrent pas l'ensemble des disciplines universitaires. On peut même ajouter que cela n'est pas obligatoirement souhaitable. II ne s'agit donc que d'une situation théorique construite pour la comparer à la réalité du public de chaque site.

Comment ce « public attendu » est-il construit ?

Continuons à raisonner sur notre exemple des sciences économiques en Aquitaine. Le public attendu de "Bordeaux campus " est l'ensemble des étudiants dont les parents résident dans le département de la Gironde. Ils sont 251. Parmi ces étudiants, $61,4 \%$ sont « à l'heure ", c'est-à-dire qu'ils ont poursuivi une scolarité sans accroc dans le secondaire, $91,3 \%$ ont un baccalauréat général, $43,4 \%$ sont des filles et $50 \%$ ont des parents cadres supérieurs ou profession libérale. Nous avons là, par construction, le public attendu en Sciences économiques à Bordeaux. Quelles sont les caractéristiques du public observé ? 66,6 \% des étudiants sont à l'heure, ce qui fait un «gain » de $5 \%$ d'étudiants de cette catégorie. $92,6 \%$ ont un baccalauréat général (+ $1 \%), 47,7 \%$ sont des filles $(+4 \%)$ et $48 \%$ sont de milieu cadre $(-2 \%)$. En d'autres termes, Bordeaux en Sciences économiques " attire " plutôt des étudiants à l'heure, détenteurs d'un baccalauréat général et de sexe féminin. Voyons ce qu'il en est pour les autres sites.

Les transferts d'étudiants d'un département à l'autre, ou d'un site à l'autre dans un même département, ont des conséquences variables sur le public étudiant. Globalement, le tableau III montre des soldes positifs pour les " bons » étudiants à Bordeaux et à $\mathrm{Pau}$ (deux sites de plein exercice), et un solde nettement négatif pour le site délocalisé de Bayonne. Les étudiants « à l'heure » devraient représenter près de $65 \%$ des effectifs dans ce site, ils ne sont que $53 \%$. De même la "perte » de bacheliers généraux est très forte, alors que Pau gagne environ $9 \%$ de ces bacheliers par rapport à l'attendu. Le tableau IV résume ces résultats.

Tableau III. - Sciences économiques et gestion.

Public attendu et observé de chaque site en Aquitaine

\begin{tabular}{|l|c|c|c|c|c|c|}
\hline & $\begin{array}{c}\text { Attendu } \\
\text { Bordeaux }\end{array}$ & $\begin{array}{c}\text { Observé } \\
\text { Bordeaux }\end{array}$ & $\begin{array}{c}\text { Attendu } \\
\text { Pau }\end{array}$ & $\begin{array}{c}\text { Observé } \\
\text { Pau }\end{array}$ & $\begin{array}{c}\text { Attendu } \\
\text { Bayonne }\end{array}$ & $\begin{array}{c}\text { Observé } \\
\text { Bayonne }\end{array}$ \\
\hline Heure & 61,4 & 66,6 & 64,7 & 71,4 & 64,7 & 53,4 \\
\hline Baccalauréat & 91,3 & 92,6 & 87,2 & 96,1 & 87,2 & 71,7 \\
\hline Filles & 43,4 & 47,7 & 54,9 & 56,2 & 54,9 & 50 \\
\hline Cadres & 50,2 & 48 & 45,2 & 50,5 & 45,2 & 28,3 \\
\hline
\end{tabular}

Tableau IV. - Sciences économiques et gestion.

Différences entre le public attendu et observé dans chaque site (en \%)

\begin{tabular}{|l|c|c|c|}
\hline & Bordeaux Campus & Pau & Bayonne \\
\hline Gain étudiants à l'heure & +5 & +9 & -12 \\
\hline Gain bacheliers généraux & +1 & +1 & -15 \\
\hline Gain étudiante & +4 & +5 & -5 \\
\hline Gain enfants de cadres & -2 & -17 \\
\hline
\end{tabular}




\section{L'ATTRACTIVITÉ DES SITES UNIVERSITAIRES}

L'attractivité des sites peut se mesurer au moins de deux façons. D'abord quantitativement, en fonction de la capacité d'un site à garder les étudiants de son département et à attirer des étudiants d'autres départements ou académies. Cette attractivité est probablement liée à l'ancienneté des sites, leur statut et leur taille. Ensuite qualitativement, on peut se questionner sur les caractéristiques des étudiants qui sont " attirés » et en quoi cela peut-il transformer la nature du public de chaque site. En d'autres termes, sur l'ensemble de l'académie, les sites attractifs attirent-ils plutôt des étudiants scolairement bons, socialement plus aisés, etc. ?

Le tableau $\mathrm{V}$ (page suivante) donne à voir une comparaison générale entre le nombre théorique et le nombre observé d'étudiants de chaque site en Aquitaine. Quelle que soit la discipline et le département, les sites délocalisés «perdent " des étudiants alors que les sites de plein exercice en gagnent. Cela signifie que les premiers fonctionnent véritablement comme un service public de proximité : ils ne recrutent pas en dehors d'une zone géographique restreinte. Mais bien plus encore, certains étudiants qui pourraient s'y inscrire préfèrent étudier dans des sites de plein exercice. Ces derniers ont pour vocation de recruter de façon bien plus large. En fonction de l'état de l'offre de formation dans l'académie, ils peuvent doubler leurs effectifs, comme en STAPS et dans une moindre mesure en Lettres et Arts, ou simplement accroitre leurs effectifs d'un tiers comme en Droit ou l'offre de formation est la plus étendue en Aquitaine en nombre de sites.

II faut se garder d'interpréter ce tableau comme on le ferait à propos d'établissements du second degré. Rappelons que le fait de "perdre " ou de "gagner" des étudiants dépend de facteurs très divers, et notamment du nombre de sites d'enseignement pour une discipline donnée. C'est ainsi que les STAPS en Aquitaine attirent toujours plus d'étudiants que prévu. Cela est lié à la situation particulière du site de Tarbes qui est l'émanation de l'université de Pau mais se situe dans les Hautes-Pyrénées. II attire donc naturellement un nombre important d'étudiants hors département et hors académie.

On peut lire ces premiers résultats comme le fruit d'une division du travail entre les sites délocalisés et de plein exercice. L'une des fonctions des délocalisations universitaires a été, dans le contexte d'accroissement des effectifs jusqu'au milieu des années
1990 , de « désengorger » les sites centraux dont les effectifs ne cessaient de croître. II semble que cette fonction se perpétue, même si les sites de plein exercice gardent un caractère attractif lié pour une part aux perspectives d'études qu'ils proposent, et pour une autre part à leur situation dans des pôles universitaires qui offrent tous les avantages des modes de vie urbains aux étudiants (Dubet et al., 1994). Le cas du Droit est de ce point de vue emblématique : les cinq sites aquitains permettent au site bordelais de n'accroître que de $34 \%$ ses effectifs par rapport à la situation théorique, alors que les Sciences de la matière et les Sciences de la vie connaissent un déséquilibre plus grand lié au faible nombre de sites délocalisés. C'est ainsi que Bordeaux en sciences de la matière reçoit 359 étudiants $(+56 \%)$ de plus que le potentiel du département pourrait le laisser croire, et en Sciences de la vie 219 de plus (+67\%).

Les sites délocalisés fonctionnent donc bien comme des sites de proximité, mais cela n'exclut pas des stratégies plus élaborées des étudiants qui peuvent choisir, soit pour des raisons universitaires, soit liées aux modes de vie urbains qu'offrent les grandes métropoles régionales, soit encore dans la perspective de prendre leur indépendance visà-vis de leurs parents, de rejoindre un site de plein exercice.

C'est ainsi que, dans le contexte de baisse des effectifs universitaires qui prévaut depuis le milieu des années 1990, cette division du travail entre les sites peut facilement devenir une concurrence. En effet, dans les disciplines telles que les sciences, mais aussi dans une moindre mesure en Droit et en Lettres, la baisse des inscriptions en première année implique de repenser la relation des sites délocalisés avec leur université de référence. Car dans ce cadre, les sites universitaires doivent se partager les étudiants (Graphique 1), jouant un « jeu à somme nulle » : ce que gagne l'un, l'autre le perd.

Les sites délocalisés ne font pas le plein ! Bayonne, Agen, Périgueux ont moins d'étudiants qu'ils ne devraient en avoir, et Bordeaux draine beaucoup plus d'étudiants que ne le laisse penser son «bassin d'inscription». Tout semble indiquer une situation de concurrence entre les sites, qui est d'autant plus forte que la proximité géographique des sites est grande. Le « derby " entre Pau et Bayonne en Droit et en Économie empêche Pau de faire le plein dans ces deux disciplines. Bordeaux, pour sa part, n'a pas ce problème puisque aucun site délocalisé n'existe en Gironde. Plus généralement, c'est toujours dans 
Tableau V. - Nombre d'étudiants "théorique " et " observé " en Aquitaine en fonction de la discipline et du site

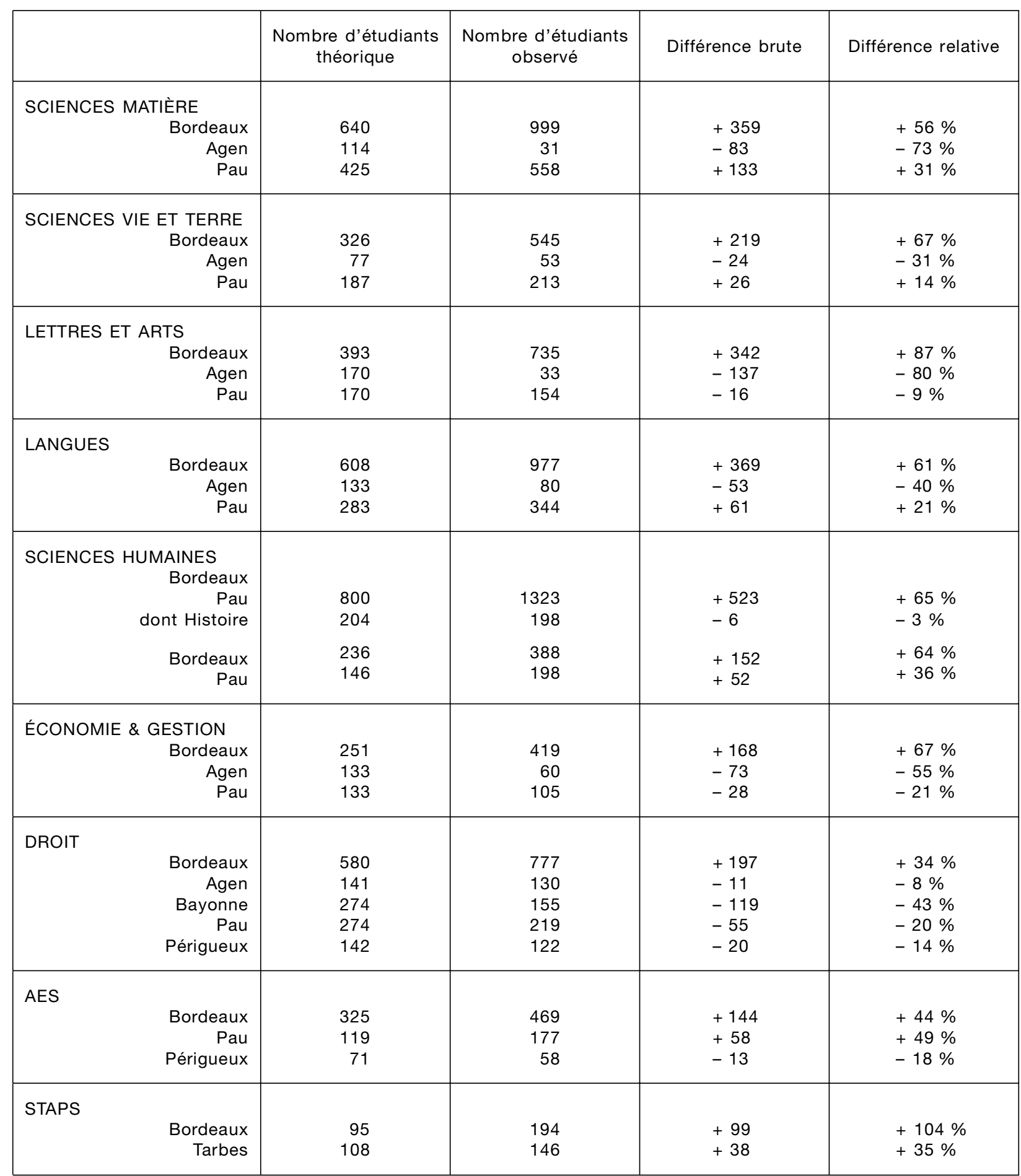




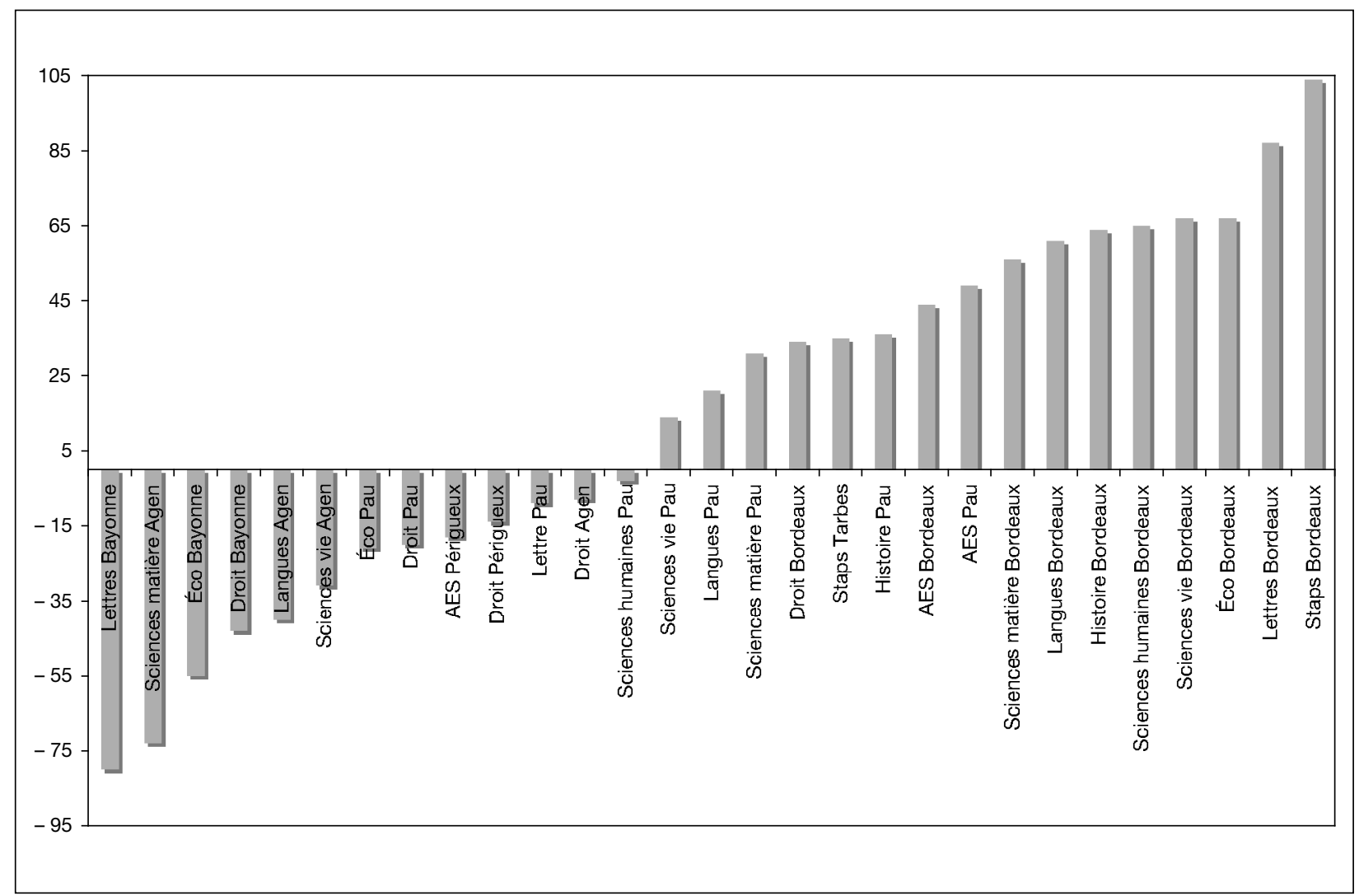

la métropole régionale que le nombre d'étudiants est supérieur à l'attendu. La question est donc de savoir comment cette attractivité se construit.

\section{LES VOIES DE L'ATTRACTIVITÉ}

On voit donc que ce qui pouvait apparaître comme un rééquilibrage de la répartition des populations étudiantes dans un contexte d'accroissement des effectifs, prend les formes d'une concurrence lorsque les inscriptions tendent à baisser. On peut alors faire l'hypothèse d'un processus assez proche de celui observé dans l'enseignement secondaire. Les établissements (ou ici les sites) les plus attractifs ont un public scolairement meilleur que l'attendu, ce qui est déjà un « effet d'établissement " puisque la nature du site transforme la nature même du public étudiant en première année.
Toutefois, avant d'aborder la question des conséquences de cette attractivité différentielles des sites, observons comment se construit cette attractivité. Elle est d'abord définie par la capacité d'un site à inscrire les étudiants de son "bassin d'inscription ", elle dépend ensuite de son attractivité sur les étudiants des autres départements, voire des autres académies.

Le taux de recrutement local est une mesure de l'attractivité des sites universitaires. II distingue fortement les sites délocalisés des sites centraux. Ceux dont le public étudiant est circonscrit dans le département joue un rôle de "sites de proximité ». C'est le cas des antennes délocalisées, qui semblent pleinement accomplir leur mission. Qu'il s'agisse de Périgueux, d'Agen ou encore de Bayonne, le taux de recrutement local frôle les $100 \%$. Seul le site de Tarbes en STAPS fait exception, ce qui s'explique par sa situation particulière. Pour le reste, ce sont les 
Graphique 2. - Taux de recrutement local en fonction de la filière et du site

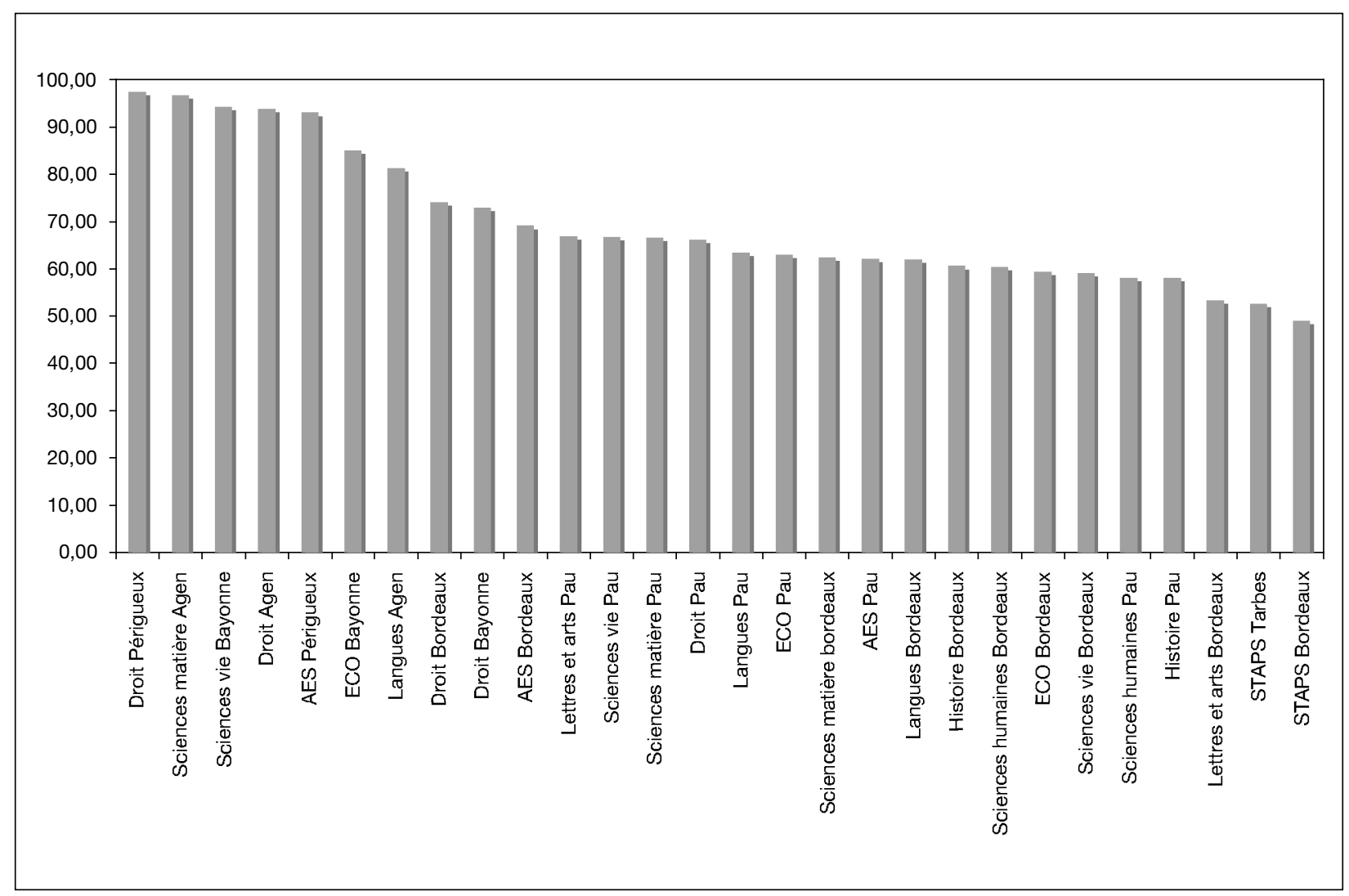

sites de plein exercice qui, en proportion, recrutent le moins localement. Leur vocation à rayonner sur l'ensemble de l'Aquitaine, voire vers les académies limitrophes, s'exprime là pleinement. Leur recrutement local oscille autour de $60 \%$. Le reste constitue un apport hors département ou hors académie.

Le taux de rétention locale mesure la capacité des sites à garder les étudiants de leur bassin de recrutement. Les taux de $100 \%$ associés au site bordelais ne doit pas faire illusion. Les bacheliers girondins qui changent d'académie échappent ici à la mesure. Toutefois, le graphique 3 montre la capacité du site bordelais à garder ses étudiants (et non pas obligatoirement ses bacheliers) : le taux de rétention locale oscille entre $95 \%$ et $100 \%$. Celui ou celle qui projette de s'inscrire en Lettres, Économie ou encore en Droit et dont les parents habitent la Gironde s'inscrit à Bordeaux dans l'immense majorité des cas. Pour les autres sites, et notamment les délocalisés, le gra- phique 3 confirme les résultats établis plus haut. Ces sites ne font pas le « plein »: le fait d'habiter le Lot-etGaronne ne signifie pas une inscription automatique à Agen par exemple. Un nombre non négligeable d'étudiants choisissent les «vraies " universités centrales : celle de Bordeaux ou de Pau.

Le fait d'attirer des étudiants venant d'une autre académie dépend semble-t-il de plusieurs facteurs. L'attractivité et la réputation ne semblent pas être déterminants, tout au moins au niveau des premiers cycles. Bien plus que cette dimension subjective, c'est la géographie qui dicte ici sa logique. Les sites de plein exercice situés dans les zones limitrophes en Aquitaine drainent un taux d'étudiants hors académie important. C'est le cas de Pau, quelle que soit la discipline. Bordeaux vient nettement derrière Pau pour ce qui est du recrutement de ce type d'étudiants. Enfin les sites délocalisés n'attirent aucun (ou presque) étudiants de ce type, sauf Tarbes par sa situation géographique et institutionnelle singulière. 
Graphique 3. - Taux de rétention locale en fonction de la discipline et du site

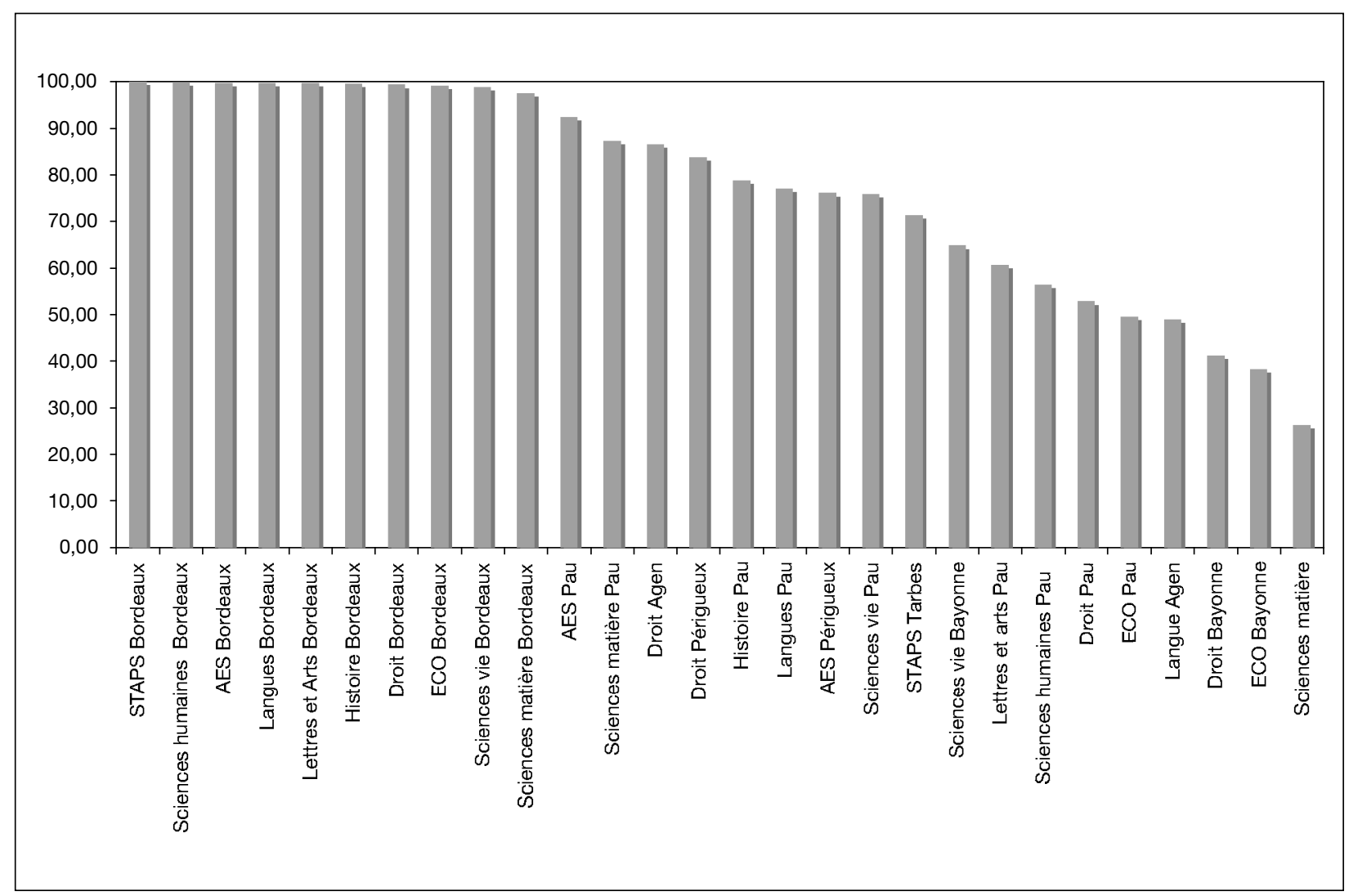

L'attractivité des sites dépend, on le voit, de facteurs aussi divers que le statut (délocalisé ou de plein exercice), la situation géographique, la taille et la discipline enseignées. De ce point de vue, le maillage territorial est ici de première importance et l'on ne peut raisonner sur les sites universitaires comme on raisonne sur les collèges et les lycées. L'attractivité d'un site semble à la fois le fruit de stratégies d'étude et de la recherche de modes de vie urbains fortement marqués par la vie estudiantine (Galland, 1996). II n'est donc pas exclu que ces transferts d'inscription soient plus volontiers le fait d'étudiants ayant des caractéristiques sociales et scolaires particulières. Le fait par exemple d'étudier dans un site délocalisé est plus souvent le choix d'étudiants académiquement faibles et de milieu modeste et populaire. C'est bien là une des sources de légitimité de ces sites: permettre à des étudiants qui ne se seraient pas inscrits dans une «grande » université, de le faire dans un site de proximité. On peut rendre compte de ce phénomène à partir des catégories du rational choice (Boudon, 1973). En effet, l'attractivité des sites universitaires apparaît comme la résultante de deux choix distincts pour les étudiants. D'abord celui d'effectuer des études longues, ensuite celui du site d'étude. Le premier choix renvoie à un calcul simple de « rendement » des études qui lui-même dépend du coût et des bénéfices escomptés en cas de réussite. Or, les filières délocalisées permettraient à ceux qui doutent de leur chance de réussir - id est les étudiants académiquement les plus faibles - de réduire le coût d'une inscription. Peuvent s'inscrire alors dans les sites délocalisés des étudiants qui, sans cette opportunité, ne se seraient pas inscrits en première année d'université. Pour les autres, plus souvent à l'heure et de milieu aisé, le coût relatif des études est plus faible et le risque d'échec est moins grand. De ce fait, leurs probabilités de faire des études longues sont plus élevées et ils s'inscrivent plus volontiers dans les sites centraux, où l'on prépare aux diplômes de licence et de master. 


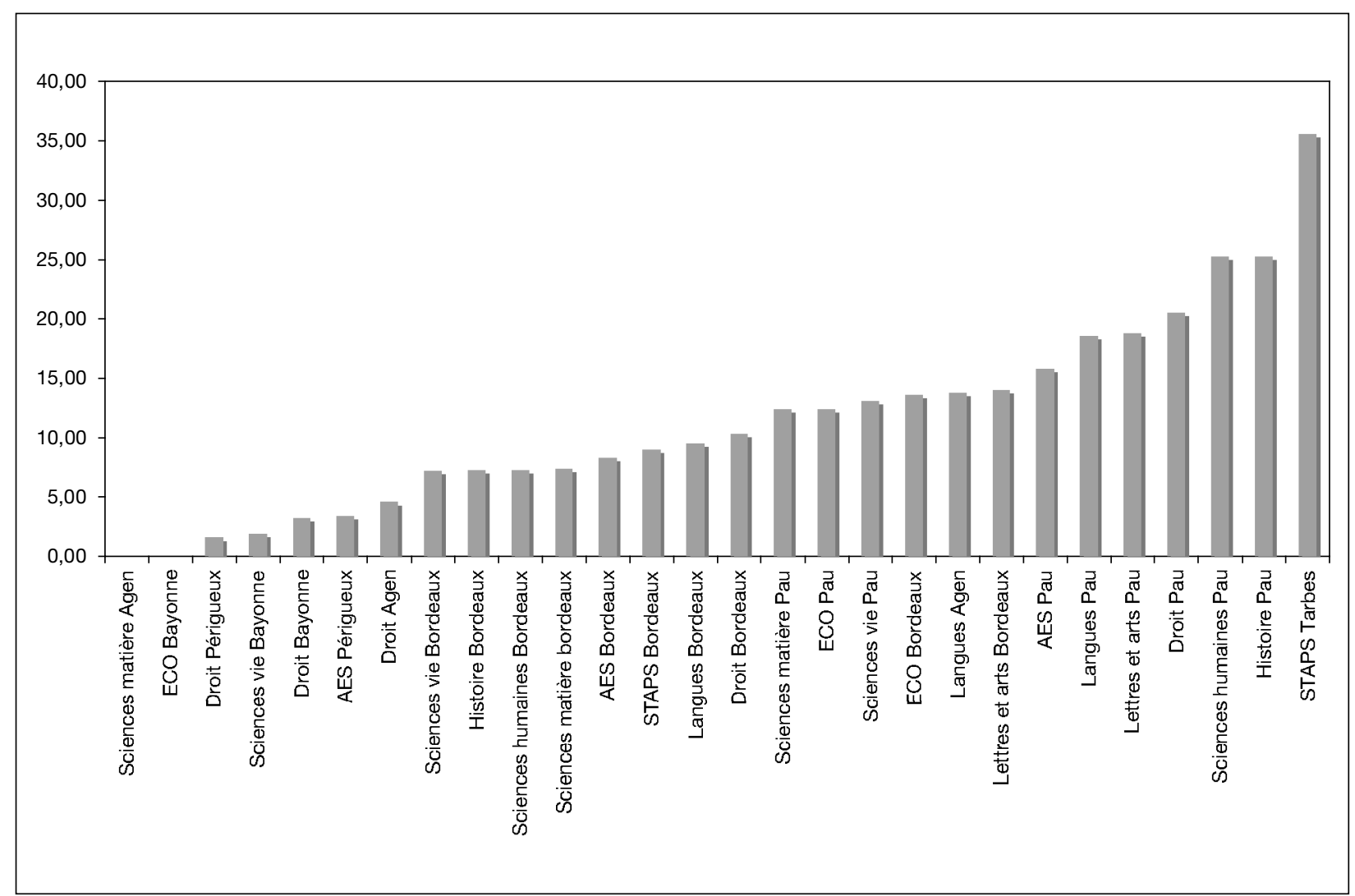

II est alors pertinent de se questionner sur les conséquences de ces choix par les étudiants. Les taux de recrutement local et de rétention montrent des disparités considérables. II est temps d'en analyser les conséquences en termes de recrutement social et scolaire dans chaque site.

\section{LES CONSÉQUENCES SUR LA NATURE DU PUBLIC ÉTUDIANT}

On connaît pour chaque site sa population « théorique " et ses caractéristiques en fonction de l'âge, de la série du bac, du sexe et de l'origine sociale. On connaît aussi sa population réelle en fonction de ces variables. II nous reste à comparer les deux pour mesurer les transformations du public de chaque site liées aux choix d'inscription des étudiants. Ces informations sont consignées dans le tableau VI. À chaque site est associé les indicateurs d'attractivité, la composition sociale et scolaire du son public et le gain lié à son attractivité. Par exemple en Sciences de la matière à Bordeaux, on observe un taux d'étudiants à l'heure de $77 \%$. Le caractère attractif de ce site fait qu'il gagne $5 \%$ d'étudiants à l'heure. S'il se contentait des étudiants de son "bassin de recrutement », ils ne seraient que $72 \%$. Son " gain » en étudiants bacheliers généraux est de $+2 \%$, sa population est stable en fonction du sexe et il « perd " $5 \%$ d'étudiants de milieu cadre. On voit que les effets de l'attractivité sont dans la plupart des cas relativement faibles, surtout dans les sites de plein exercice. Pour les sites délocalisés, l'amplitude atteint plus de $15 \%$, ce qui laisse penser que le public de ces sites est fortement marqué par leur mode de recrutement.

La lecture du tableau VI montre des phénomènes somme toute prévisibles: les meilleurs étudiants, 
Tableau VI. - Modes de recrutement par sites et filières et conséquences sur le public étudiant (4)

\begin{tabular}{|c|c|c|c|c|c|c|c|c|c|c|c|}
\hline & 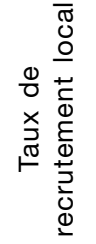 & 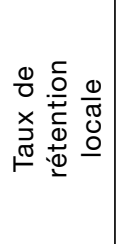 & 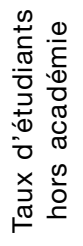 & 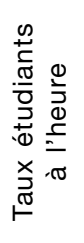 & 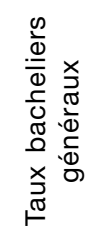 & 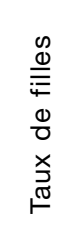 & 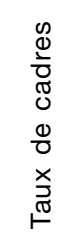 & 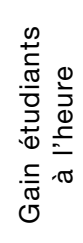 & 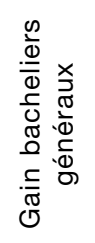 & 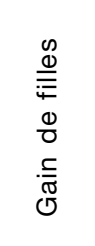 & 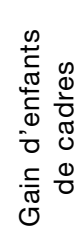 \\
\hline $\begin{array}{r}\text { SCIENCES MATIÈRE } \\
\text { ET MIASS } \\
\text { Bordeaux Campus } \\
\text { Agen } \\
\text { Pau }\end{array}$ & $\begin{array}{l}62,50 \\
96,80 \\
66,50\end{array}$ & $\begin{array}{l}97,5 \\
26,3 \\
87,3\end{array}$ & $\begin{array}{c}7,4 \\
0 \\
12,4\end{array}$ & 66,9 & 82,4 & 42,6 & $\begin{array}{l}48,5 \\
35,3\end{array}$ & $\begin{array}{l}+5 \\
-4\end{array}$ & $\begin{array}{l}+2 \\
-3\end{array}$ & $\begin{array}{c}0 \\
+1,5\end{array}$ & $\begin{array}{l}-5 \\
-4\end{array}$ \\
\hline $\begin{array}{r}\text { SCIENCES DE LA } \\
\text { VIE ET TERRE } \\
\text { Bordeaux Campus } \\
\text { Agen } \\
\text { Pau }\end{array}$ & $\begin{array}{l}59,1 \\
94,3 \\
66,7\end{array}$ & $\begin{array}{l}98,8 \\
64,9 \\
75,9\end{array}$ & $\begin{array}{c}7,2 \\
1,9 \\
13,1\end{array}$ & $\begin{array}{c}64 \\
60,4 \\
60,6\end{array}$ & $\begin{array}{c}99,3 \\
100,00 \\
94\end{array}$ & $\begin{array}{c}48,8 \\
51 \\
56,8\end{array}$ & $\begin{array}{l}44,2 \\
41,5 \\
33,7\end{array}$ & $\begin{array}{l}+4 \\
-8 \\
-3\end{array}$ & $\begin{array}{c}0 \\
+1 \\
-1\end{array}$ & $\begin{array}{c}0 \\
-4 \\
0\end{array}$ & $\begin{array}{l}-1 \\
+5 \\
-7\end{array}$ \\
\hline $\begin{array}{r}\text { LETTRES ET ARTS } \\
\text { Bordeaux Campus } \\
\text { Bayonne } \\
\text { Pau }\end{array}$ & $\begin{array}{l}53,3 \\
75,8 \\
66,9\end{array}$ & $\begin{array}{l}99,7 \\
14,7 \\
60,6\end{array}$ & $\begin{array}{c}14 \\
3 \\
18,8\end{array}$ & $\begin{array}{l}61,2 \\
69,5\end{array}$ & $\begin{array}{c}96 \\
98,6\end{array}$ & $\begin{array}{l}76,6 \\
83,1\end{array}$ & $\begin{array}{l}36,1 \\
28,4\end{array}$ & $\begin{array}{l}+5 \\
+5\end{array}$ & $\begin{array}{c}+0,5 \\
0\end{array}$ & $\begin{array}{l}-2 \\
+1\end{array}$ & $\begin{array}{l}-4,5 \\
-1,5\end{array}$ \\
\hline $\begin{array}{l}\text { LANGUES } \\
\text { Bordeaux Campus } \\
\text { Agen } \\
\text { Pau }\end{array}$ & $\begin{array}{c}62 \\
81,3 \\
63,4\end{array}$ & $\begin{array}{c}99,7 \\
48,9 \\
77\end{array}$ & $\begin{array}{c}9,5 \\
13,8 \\
18,6\end{array}$ & $\begin{array}{c}60 \\
56,2 \\
62,5\end{array}$ & $\begin{array}{c}91 \\
86,1 \\
91,9\end{array}$ & $\begin{array}{c}82 \\
78,7 \\
82,8\end{array}$ & $\begin{array}{c}28,4 \\
21,1 \\
23\end{array}$ & $\begin{array}{c}+4 \\
-4 \\
-1,5\end{array}$ & $\begin{array}{c}+2 \\
-2 \\
0\end{array}$ & $\begin{array}{l}+2 \\
-2 \\
-2\end{array}$ & $\begin{array}{c}-1 \\
+1,5 \\
0\end{array}$ \\
\hline $\begin{array}{r}\text { SCIENCES HUMAINES } \\
\text { Bordeaux Campus } \\
\text { Pau } \\
\text { dont Histoire } \\
\text { Bordeaux Campus } \\
\text { Pau }\end{array}$ & $\begin{array}{l}60,4 \\
58,1 \\
\\
60,6 \\
58,1\end{array}$ & $\begin{array}{l}99,9 \\
56,4 \\
99,6 \\
78,8\end{array}$ & $\begin{array}{c}7,3 \\
25,3 \\
\\
7,3 \\
25,3\end{array}$ & $\begin{array}{c}61,1 \\
55\end{array}$ & $\begin{array}{c}84,4 \\
90,5 \\
\\
91 \\
90,5\end{array}$ & $\begin{array}{l}69,4 \\
54,5 \\
53,6 \\
54,5\end{array}$ & $\begin{array}{l}28,5 \\
23,8 \\
35,1 \\
23,8\end{array}$ & $\begin{array}{l}+2,5 \\
+2,5 \\
+4 \\
+1\end{array}$ & $\begin{array}{l}+1 \\
+3 \\
+4 \\
+1\end{array}$ & $\begin{array}{l}+2 \\
-8 \\
0 \\
+1\end{array}$ & $\begin{array}{l}-1 \\
-2 \\
\\
0 \\
-3\end{array}$ \\
\hline $\begin{array}{r}\text { ÉCONOMIE } \\
\text { ET GESTION } \\
\text { Bordeaux Campus } \\
\text { Bayonne } \\
\text { Pau }\end{array}$ & $\begin{array}{c}59,4 \\
85 \\
62,9\end{array}$ & $\begin{array}{l}99,2 \\
38,3 \\
49,6\end{array}$ & $\begin{array}{c}13,6 \\
0 \\
12,4\end{array}$ & $\begin{array}{l}66,6 \\
53,4 \\
71,4\end{array}$ & $\begin{array}{l}92,6 \\
71,7 \\
96,1\end{array}$ & $\begin{array}{c}47,7 \\
50 \\
56,2\end{array}$ & $\begin{array}{c}48 \\
28,3 \\
50,5\end{array}$ & $\begin{array}{c}+5 \\
-12 \\
+6\end{array}$ & $\begin{array}{c}+1 \\
-15 \\
+9\end{array}$ & $\begin{array}{l}+4 \\
-5 \\
+1\end{array}$ & $\begin{array}{c}-2 \\
-17 \\
+5\end{array}$ \\
\hline $\begin{array}{l}\text { DROIT } \\
\text { Bordeaux Campus } \\
\text { Agen } \\
\text { Bayonne } \\
\text { Pau } \\
\text { Périgueux }\end{array}$ & $\begin{array}{l}74,1 \\
93,8 \\
72,9 \\
66,2 \\
97,5\end{array}$ & $\begin{array}{l}99,3 \\
86,5 \\
41,2 \\
52,9 \\
83,8\end{array}$ & $\begin{array}{c}10,3 \\
4,6 \\
3,2 \\
20,5 \\
1,6\end{array}$ & $\begin{array}{l}57,2 \\
44,6 \\
45,2 \\
57,1 \\
52,5\end{array}$ & $\begin{array}{c}87,2 \\
73 \\
74,8 \\
84,9 \\
72,1\end{array}$ & $\begin{array}{l}70,5 \\
71,5 \\
63,9 \\
74 \\
69,7\end{array}$ & $\begin{array}{l}42,4 \\
16,9 \\
23,3 \\
31,5 \\
17,2\end{array}$ & $\begin{array}{c}0 \\
-5 \\
-7,5 \\
+4,5 \\
-2\end{array}$ & $\begin{array}{l}+1 \\
-2 \\
-4 \\
+6,5 \\
-4,5\end{array}$ & $\begin{array}{l}0 \\
0 \\
-3 \\
+7 \\
+1\end{array}$ & $\begin{array}{l}-1 \\
0 \\
-6,5 \\
+1 \\
-1,5\end{array}$ \\
\hline $\begin{array}{l}\text { AES } \\
\text { Bordeaux Campus } \\
\text { Pau } \\
\text { Périgueux }\end{array}$ & $\begin{array}{l}69,1 \\
62,1 \\
93,1\end{array}$ & $\begin{array}{l}99,7 \\
92,4 \\
76,1\end{array}$ & $\begin{array}{c}8,3 \\
15,8 \\
3,4\end{array}$ & $\begin{array}{l}40,7 \\
44,6 \\
47,8\end{array}$ & $\begin{array}{l}74,2 \\
70,1 \\
86,1\end{array}$ & $\begin{array}{l}58,2 \\
62,7 \\
58,6\end{array}$ & $\begin{array}{c}24 \\
17,5 \\
22,5\end{array}$ & $\begin{array}{c}0 \\
+5 \\
-4\end{array}$ & $\begin{array}{l}-3 \\
+4 \\
+4\end{array}$ & $\begin{array}{c}0 \\
+1,5 \\
+2\end{array}$ & $\begin{array}{c}0 \\
+2 \\
-3\end{array}$ \\
\hline $\begin{array}{l}\text { STAPS } \\
\text { Bordeaux Campus } \\
\text { Tarbes }\end{array}$ & $\begin{array}{c}49 \\
52,7\end{array}$ & $\begin{array}{c}100 \\
71,3\end{array}$ & $\begin{array}{c}9 \\
35,6\end{array}$ & $\begin{array}{l}52 \\
39\end{array}$ & $\begin{array}{c}79,4 \\
87\end{array}$ & $\begin{array}{l}36,6 \\
34,9\end{array}$ & $\begin{array}{l}36,6 \\
28,1\end{array}$ & $\begin{array}{l}2,5 \\
-6\end{array}$ & $\begin{array}{c}-0,5 \\
-3\end{array}$ & $\begin{array}{l}-2 \\
-2\end{array}$ & $\begin{array}{c}+2 \\
-0,5\end{array}$ \\
\hline
\end{tabular}

* Effectifs réduits (inférieurs à 30 individus). 
Graphique 5. - Gain des sites en étudiants à l'heure

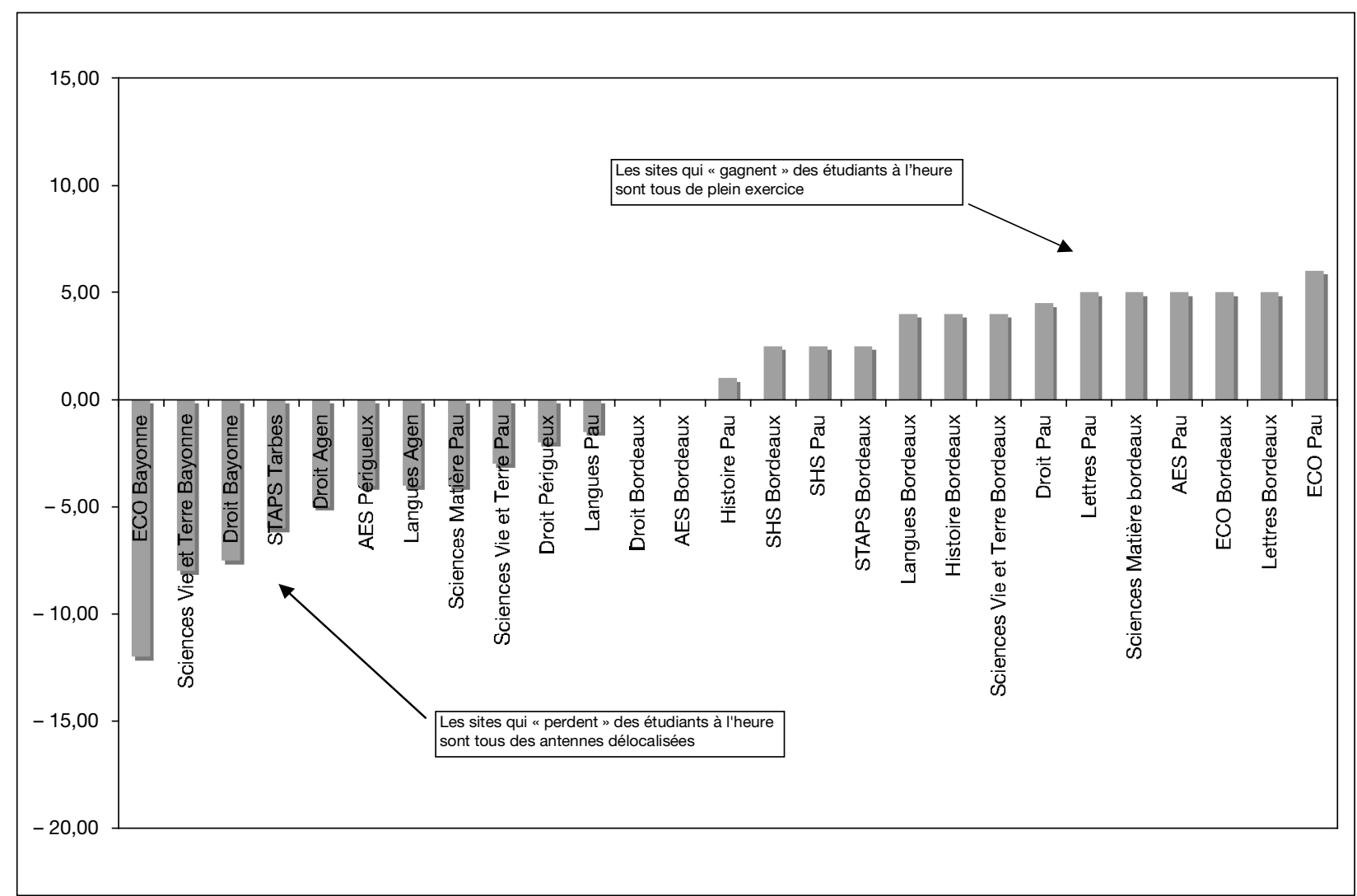

notamment les moins âgés en première année, rejoignent plus volontiers les sites centraux que les sites délocalisés (Graphique 4). Tous les sites délocalisés " perdent » des étudiants à l'heure alors que ceux de plein exercice en gagnent systématiquement. Très souvent, le gain des sites centraux est dû à l'apport des étudiants hors académie car les «migrations" étudiantes sont plus souvent le fait des bons étudiants plutôt que des plus faibles. Inversement les délocalisés ne reçoivent que rarement des étudiants hors département, ce qui limite l'apport potentiel d'étudiants à l'heure.

Les sites qui inscrivent bien moins d'étudiants à I'heure que prévu sont d'abord Bayonne (en Économie, Sciences de la vie et Droit), Tarbes (en STAPS), Agen et Périgueux. Ces sites semblent se «spécialiser » en étudiants peu performants, alors que Pau et Bordeaux ont un public plus jeune que prévu. Les chiffres présentés au tableau 6 le montrent: les sites délocalisés ont systématiquement moins d'étudiants à l'heure que les sites centraux, quelle que soit la discipline. En Sciences économiques et Gestion par exemple, seulement $53 \%$ des étudiants de Bayonne sont "à l'heure ", contre 66,6 \% à Bordeaux et plus de $71 \%$ à Pau. De même en droit, on passe de $44,6 \%$ à Agen à $57 \%$ à Bordeaux et à Pau.

La relation entre attractivité et nature du public inscrit dans chaque site est moins claire en ce qui concerne les bacheliers généraux, et ceci pour deux raisons. D'abord les taux sont très élevés (le plus faible taux est de $70 \%$ en AES à Pau), ce qui limite nécessairement les variations qui ne peuvent dépasser les $100 \%$. Ensuite, la série du baccalauréat dépend fortement de la discipline suivie. Certaines spécialités, y compris en sciences, admettent des étudiants dont le baccalauréat n'est pas général, ce qui génère du «bruit » dans la lecture que l'on peut faire des données du graphique 5 . 
Graphique 6. - Gain des sites en bacheliers généraux

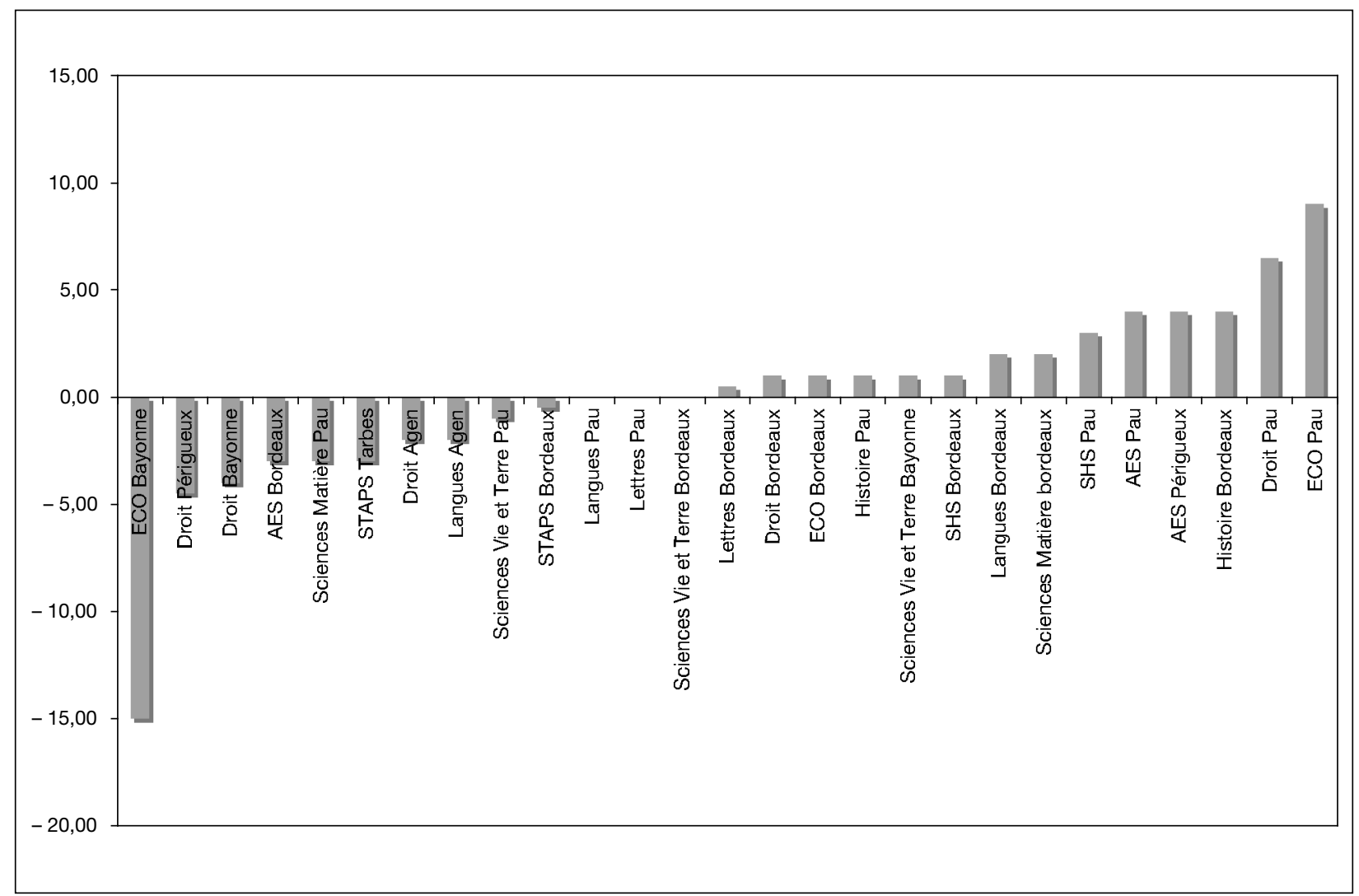

AES à Bordeaux perd des bacheliers généraux, mais très faiblement $(-3 \%)$ alors que Périgueux en gagne $5 \%$. De même, sciences de la matière à Pau est dans ce cas. Mais les écarts restent faibles, sauf pour l'Économie et la Gestion à Bayonne (- $15 \%$ ) qui semble nettement profité à Pau (+9\%). Dans ce cas, la « division du travail » entre le site central de Pau et le délocalisé de Bayonne semble claire. Le premier attire les meilleurs étudiants, le second ne garde que les moins dotés. Cela s'observe tant pour les étudiants à l'heure, que pour les bacheliers généraux ou encore les étudiants de milieu cadre. Doit-on, dans ce cas, parler de "concurrence " ou de répartition des tâches? Les deux hypothèses ne sont pas ici contradictoires. Les sites délocalisés ont une fonction de proximité inscrite dans leur situation même. II n'empêche qu'ils se font " piquer » une part de leurs meilleurs étudiants par les «vraies » universités centrales. On peut ajouter que le même processus est à l'œuvre pour les études de droit. Périgueux, Agen et
Bayonne tendent à perdre de «bons » étudiants au profit de Pau et de Bordeaux.

L'attractivité des sites agit moins sur la répartition en fonction du sexe. L'amplitude est bien plus faible que pour les variables plus proprement liées au niveau académique des étudiants, tels que l'âge et la série du baccalauréat. Toutefois, les sites centraux tendent à gagner des étudiantes, sauf pour les disciplines scientifiques. Pour les étudiants de milieu cadre, les effets de l'attractivité sont complexes à interpréter car ils dépendent nettement de la discipline. Dans la plupart des cas, les différences sont inexistantes ou minimes. Ce sont les sciences économiques qui se détachent du lot: Bayonne est «fui » par ces étudiants et Pau en récolte les bénéfices.

En définitive, la nature de l'offre de formation dans une discipline donnée en Aquitaine influe nettement sur la composition sociale et académique des différents sites. La comparaison entre le public « attendu » 
et le public « observé » montre de nettes différences qui reflètent une division du travail entre les sites centraux et leurs antennes délocalisées. Très souvent, la vocation de «proximité " de ces sites délocalisés est renforcée par les stratégies des meilleurs étudiants qui choisissent plus souvent que les autres de rejoindre les sites de plein exercice. Les sites les plus attractifs, id est qui recrutent au-delà de leur département, sont aussi ceux qui gagnent le plus d'étudiants «à l'heure ". Dès lors que le taux de recrutement local se situe entre $60 \%$ et $70 \%$, le gain en « bons " étudiants oscille autour de $5 \%$. Cette relation n'est pas systématique. Elle dessine toutefois une réelle tendance des sites de plein exercice à attirer plutôt de bons éléments. Les conséquences de ces « transferts " ne sont pas négligeables car cela renforce la tonalité sociale et académique de chaque site. Dès lors que l'offre de formation universitaire se diversifie pour une discipline donnée, le public étudiant en première année tend, lui aussi, à se diversifier en fonction du site d'étude. Les délocalisations procéderaient alors d'un double processus. Celui d'une démocratisation du recrutement en première année, tant du point de vue social que scolaire. Celui aussi, d'une différenciation et d'une spécialisation dans l'inscription des catégories d'étudiants socialement et scolairement les moins dotés.

\section{CONCLUSION}

Les résultats présentés donnent à voir un paysage universitaire à la fois touffu et contrasté. Touffu car le nombre des sites qui proposent un enseignement de premier cycle en Aquitaine est important. Des villes moyennes telles que Mont-de-Marsan (un IUT, des premiers cycles), Bayonne ou encore Agen, offrent des formations qui n'existaient pas il y a seulement quinze ans. Contrasté, car les conditions d'étude comme la nature du public distinguent fortement les sites délocalisés des sites centraux. Nous avons de plus montré que les choix d'inscription des étudiants, pour une discipline donnée, tend à accentuer ces contrastes : les sites délocalisés «perdent» des étudiants à I'heure et de milieu cadre, alors que les sites centraux en « gagnent " par rapport à ce que l'on pourrait attendre. Cela signifie que, de manière quasi mécanique, l'augmentation de l'offre de formation dans une discipline, accentue la différenciation sociale et scolaire des publics des premiers cycles. Tout en démocratisant l'accès aux premiers cycles, en favorisant l'inscription d'étudiants qui sans cela ne se seraient probablement pas inscrits, cet élargissement de l'offre accentue les différences dans la nature des publics de première année. Chaque site devient plus « homogène " en attirant tel ou tel type d'étudiant. Nous observons donc un double processus de démocratisation d'une part, et de différenciation d'autre part. Et cela questionne la mise en œuvre des politiques universitaires, tant au niveau national que local. Si le but de ces politiques est d'élargir la répartition territoriale des formations supérieures, le pari est réussi. Dans le domaine de la démocratisation de l'accès à l'enseignement supérieur, là encore on observe un net élargissement du recrutement social des premiers cycles et l'on s'accorde pour dire que cet élargissement est un moyen de démocratiser les études supérieures, domaine dans lequel la France a encore quelques progrès à faire par rapport à ses voisins européens (OCDE, 2005). Toutefois, cet accès croissant en premier cycle des étudiants les moins favorisés se fait au prix d'une différenciation sociale et académique des sites dont on ne peut aujourd'hui mesurer toutes les conséquences en termes universitaires comme en termes de formation. À l'université comme dans l'enseignement secondaire, l'élargissement de la base sociologique du public étudiant renforce, de manière quasi mécanique, les différenciations entre filières, établissements et sites universitaires.

Georges Felouzis georges.felouzis@free.fr Université Victor Ségalen-Bordeaux 2 Laboratoire d'analyse des problèmes sociaux et de l'action collective (LAPSAC)

\section{NOTES}

(1) La recherche a été financée par la Direction de l'évaluation et de la prospective (DEP) du ministère de l'Éducation nationale et par le Commissariat général au Plan dans le cadre de l'appel d'offre "Efficacité des système d'éducation et de formation ".

(2) II s'agit du fichier qui recense l'ensemble des étudiants inscrits dans une formation universitaire chaque année. II est renseigné par les universités et est centralisé par la DEP.

(3) Nous désignons par «Bordeaux campus " l'ensemble du Campus qui s'étend dans la communauté urbaine de Bordeaux (Pessac, Mérignac et Bordeaux).
(4) Toutes les filières ne sont pas présentes sur plusieurs sites en Aquitaine. C'est pourquoi nous n'avons considéré que les grandes disciplines : Sciences de la matière et MIASS (Mathématiques et informatique appliquées au sciences sociales), Sciences de la vie et de la terre, Lettres et Arts, Langues, Sciences humaines et sociales (avec un éclairage sur l'Histoire), Économie et gestion, Droit, AES et STAPS. Certains sites n'ont que des effectifs très réduits. C'est le cas des sciences de la matière à Agen et des Lettres et Arts à Bayonne. Pour ces deux sites, certains taux n'ont pas été calculés. 


\section{BIBLIOGRAPHIE}

Boudon R. L'inégalité des chances : la mobilité sociale dans les société industrielles. Paris : A. Colin, 1973.

Bourdon F. ; Duru-Bellat M. ; Jarousse J.-P. ; Peyron C. \& RAPIAU M.-T. (1994). «Délocalisations universitaires : le cas de Nevers ", Annales de la recherche urbaine, $\mathrm{n}^{\circ}$ 62-63, p. 100-111.

Dubet F. ; Filâtre D. ; Merrien F.-X. ; Sauvage A. \& Vince A (1994). Universités et villes. Paris : L'Harmattan.

FELOUZIS G. «Repenser les inégalités à l'université : des inégalités sociales aux inégalités locales dans trois disciplines universitaires ». Sociétés contemporaines, $n^{\circ} 38$, p. 67-98.

FELouzIs G. (2001a). « Les délocalisations universitaires et la démocratisation de l'enseignement supérieur ». Revue française de pédagogie, $\mathrm{n}^{\circ} 136$, p. 53-63.

FELouzIs G. (2001b). La condition étudiante. Paris : PUF.

FILÂTRE D. (2003). " Les universités et le territoire : nouveau contexte, nouveaux enjeux ». In G. Felouzis (dir.), Les mutations actuelles de l'université. Paris : PUF, p. 19-45.
France: MINISTÈre dE L'Éducation NATIONALE: DiRECTION DE L'ÉVALUATION ET DE LA PROSPECTIVE (2003). Géographie de l'école, $\mathrm{n}^{\circ} 8$

France: MINISTÈre dE L'Éducation NATIONALE: DiRECTION DE L'ÉVALUATION ET DE LA PROSPECTIVE (2004). Note d'information, $\mathrm{n}^{\circ}$ 04-39 : «Premières estimations de la rentrée 2004 dans l'enseignement supérieur».

GALland O. (1996). Le monde des étudiants. Paris : PUF.

Grossetti M (1995). Science, industrie et territoire. Toulouse : Presses universitaires du Mirail.

Losego P. (2004). « Le travail invisible à l'université : le cas des antennes universitaires ». Sociologie du travail, vol. $46, n^{\circ} 2$, p. 187-204.

MichaUt C. (2005). «Les effets des politiques de délocalisation universitaire sur les parcours des étudiants ». In Y. Dutercq (dir.), Les régulations des politiques d'éducation. Rennes : Presses universitaires de Rennes, p. 141-150.

ORgANISATION POUR LA COOPÉRATION ET LE DÉVELOPPEMENT ÉCONOMIQUE [OCDE] (2005). Regard sur l'éducation. Paris : OCDE. 